\title{
Increased prevalence of cardiac autonomic dysfunction at different degrees of glucose intolerance in the general population: the KORA S4 survey
}

\author{
Dan Ziegler • Andreas Voss • Wolfgang Rathmann • \\ Alexander Strom • Siegfried Perz • Michael Roden • \\ Annette Peters • Christa Meisinger • for the KORA Study Group
}

Received: 15 October 2014 / Accepted: 30 January 2015 / Published online: 28 February 2015

(C) Springer-Verlag Berlin Heidelberg 2015

\begin{abstract}
Aims/hypothesis Cardiac autonomic nervous dysfunction (CAND) raises the risk of mortality, but the glycaemic threshold at which it develops is unclear. We aimed to determine the prevalence of, risk factors for and impact of CAND in glucose intolerance and diabetes.

Methods Among 1,332 eligible participants aged 55-74 years in the population-based cross-sectional KORA S4 study, 130
\end{abstract}

Electronic supplementary material The online version of this article (doi:10.1007/s00125-015-3534-7) contains peer-reviewed but unedited supplementary material, which is available to authorised users.

D. Ziegler $(\bowtie) \cdot$ A. Strom $\cdot$ M. Roden

Institute for Clinical Diabetology, German Diabetes Center at

Heinrich Heine University, Auf'm Hennekamp 65,

40225 Düsseldorf, Germany

e-mail: dan.ziegler@ddz.uni-duesseldorf.de

D. Ziegler $\cdot$ M. Roden

Department of Endocrinology and Diabetology, Medical Faculty,

University Hospital, Heinrich Heine University,

Düsseldorf, Germany

\section{A. Voss}

Department of Medical Engineering and Biotechnology, University of Applied Sciences, Jena, Germany

\section{W. Rathmann}

Institute of Biometrics and Epidemiology, German Diabetes Center at Heinrich Heine University, Leibniz Center for Diabetes Research, University Hospital, Düsseldorf, Germany

\section{S. Perz}

Institute for Biological and Medical Imaging, Helmholtz Zentrum München-German Research Center for Environmental Health,

Neuherberg, Germany

A. Peters $\cdot$ C. Meisinger

Institute of Epidemiology II, Helmholtz Zentrum München-German

Research Center for Environmental Health, Neuherberg, Germany had known diabetes mellitus (k-DM), and the remaining 1,202 underwent an OGTT. Heart rate variability (HRV) and QT variability were computed from supine 5 min ECGs.

Results In all, 565 individuals had normal glucose tolerance (NGT), 336 had isolated impaired fasting glucose (i-IFG), 72 had isolated impaired glucose tolerance (i-IGT), 151 had combined IFG-IGT (IFG-IGT) and 78 had newly detected diabetes mellitus (n-DM). Adjusted normal HRV limits were defined in the NGT population (5th and 95th percentiles). Three HRV measures were more frequently abnormal in those with k-DM, n-DM, IFG-IGT and i-IFG than in those with NGT $(p<0.05)$. The rates of CAND ( $\geq 2$ of 4 HRV indices abnormal) were: NGT, 4.5\%; i-IFG, 8.1\%; i-IGT, 5.9\%; IFG-IGT, $11.4 \%$; n-DM, $11.7 \%$; and k-DM, $17.5 \%$ ( $p<0.05$ vs NGT, except for i-IGT). Reduced HRV was associated with cardiovascular risk factors used to construct a simple screening score for CAND. Mortality was higher in participants with reduced HRV ( $p<0.05$ vs normal HRV).

Conclusions/interpretation In the general population aged 5574 years, the prevalence of CAND is increased not only in individuals with diabetes, but also in those with IFG-IGT and, to a lesser degree, in those with i-IFG. It is associated with mortality and modifiable cardiovascular risk factors which may be used to screen for diminished HRV in clinical practice.

Keywords Cardiac autonomic dysfunction . Cardiovascular risk factors - Diabetes - Heart rate variability - Impaired fasting glucose - Impaired glucose tolerance
Abbreviations
Acor
Auto-correlation function
ati
Auto-transformation function
CAN 


$\begin{array}{ll}\text { CAND } & \text { Cardiac autonomic nervous dysfunction } \\ \text { DFA } & \text { Detrended fluctuation analysis } \\ \text { HF } & \text { High frequency } \\ \text { HRV } & \text { Heart rate variability } \\ \text { (i)-IFG } & \text { (Isolated) impaired fasting glucose } \\ \text { (i)-IGT } & \text { (Isolated) impaired glucose tolerance } \\ \text { ISA } & \text { Intrinsic sympathomimetic activity } \\ \text { k-DM } & \text { Known diabetes mellitus } \\ \text { LF } & \text { Low frequency } \\ \text { n-DM } & \text { Newly detected diabetes mellitus } \\ \text { NFG } & \text { Normal fasting glucose } \\ \text { NGT } & \text { Normal glucose tolerance } \\ \text { NLD } & \text { Nonlinear dynamics } \\ \text { NN } & \text { Normal-to-normal } \\ \text { NPV } & \text { Negative predictive value } \\ \text { NU } & \text { Normalised unit } \\ \text { pNN50 } & \text { Number of NN }>50 \text { ms/number of all NN } \\ \text { pNN110 } & \text { Portion of NN differences }<10 \text { ms } \\ & \text { in all NN } \\ \text { PPV } & \text { Positive predictive value } \\ \text { QTVI } & \text { QT Variability Index } \\ \text { Renyi0.25/2/4 } & \text { Renyi entropy of the histogram with } \\ & \text { (order) } \alpha=0.25 / 2 / 4 \\ \text { RMSSD } & \text { Root mean squared successive difference } \\ \text { SDANN1 } & \text { SD of NN averages in 1 min segments } \\ \text { SDSA } & \text { SD of the short axis } \\ \text { SDLA } & \text { SD of the long axis } \\ \text { SNRI } & \text { Serotonin noradrenaline reuptake inhibitors } \\ \text { SSRI } & \text { Selective serotonin reuptake inhibitors } \\ \text { TP } & \text { Total power } \\ \text { ULF } & \text { Ultra low frequency } \\ \text { VLF } & \text { Very low frequency } \\ \text { WD } & \text { Word distribution } \\ & \end{array}$

\section{Introduction}

During the past two decades, there has been considerable interest in the relations of fasting glucose and $2 \mathrm{~h}$ post-load glucose with cardiovascular morbidity and mortality $[1,2]$. Accumulated evidence has shown that $2 \mathrm{~h}$ post-load glucose is a stronger risk predictor than fasting glucose of incident coronary heart disease and cardiovascular mortality $[3,4]$. However, whether impaired fasting glucose (IFG) and impaired glucose tolerance (IGT) are associated with a higher risk of cardiovascular autonomic neuropathy (CAN) is a matter of ongoing debate [5-7]. A limited number of studies have reported contradictory data on whether reduced heart rate variability (HRV) may be more frequently encountered in persons with IFG and IGT than in those with normal glucose tolerance (NGT) or normal fasting glucose (NFG) in representative populations [8-16]. Several relatively small clinic-based studies which were not representative for the general population were also published
[17-23]. No studies have established the normal limits for HRV variables adjusted for the most relevant confounding variables to provide the prevalence rates of abnormalities in HRV indices across the complete spectrum of glucose intolerance.

Traditionally, HRV has been quantified using indices in the time domain (statistical and geometric analysis) and frequency domain (spectral analysis). More recently, methods derived from nonlinear dynamics (NLD) have provided new insights into HRV changes in various diseases [24]. Examples for measures derived from NLD include: (1) the Poincaré plot, a quantitative visual technique, in which the shape of the plot is categorised into functional classes; (2) entropy measures which assess the regularity/irregularity or randomness of heartbeat fluctuations; and (3) symbolic dynamics which allow a simple description of the coarsegrained dynamics of HR fluctuations using a limited amount of symbols [24].

In addition to HRV, temporal fluctuations in ventricular repolarisation can be quantified by measuring beat-to-beat variability in the QT interval $[25,26]$. The QT variability index (QTVI) is a noninvasive measure of repolarisation lability and has been defined as the ratio of normalised QT variability to normalised HRV; it therefore includes an assessment of autonomic nervous system tone [25]. Higher QT variability has been found in patients with cardiovascular disease and is regarded as an indicator of cardiac sympathetic activation and predictor of mortality and sudden death [26].

In the present study, we aimed to determine the prevalence of and risk factors associated with cardiac autonomic nervous dysfunction (CAND) and its impact on mortality. We also aimed to construct a simple score to screen for CAND in clinical practice, using a comprehensive array of linear and nonlinear indices of HRV and QT variability (some of which have not been previously used) across the complete spectrum of glucose intolerance, compared with individuals with NGT and those with newly detected and known diabetes mellitus (n-DM and k-DM, respectively).

\section{Methods}

Study population The present analysis is based on the KORA S4 study, a population-based health survey conducted in the city of Augsburg and two surrounding counties between October 1999 and April 2001. The study was approved by the local ethics committee and all participants gave written informed consent. The details of the study design have been described elsewhere [27]. Briefly, a total sample of 6,640 individuals was drawn in a two-stage cluster sample from the target population consisting of all German residents of the region aged 25-74 years, 4,261 (64.2\%) of whom participated in the S4 survey. All participants aged 55-74 years $(n=2,656)$ were invited to undergo an OGTT, and $62 \%(n=1,636)$ agreed 
[27]. Among these, 304 had to be excluded because of atrial fibrillation or flutter, left and right bundle-branch block, 2nd and 3rd degree atrioventricular block or sinoatrial block, multiple supraventricular or ventricular extrasystoles, pacemaker therapy, treatment with class I antiarrhythmics or uncertain fasting state at OGTT start. The present analysis focused on the remaining 1,332 participants aged 55-74 years. Previously known diabetes was defined on the basis of a self-reported physician diagnosis or the use of glucoselowering drugs. Diabetes was known in 130 of the 1,332 participants; the remaining 1,202 volunteers underwent an OGTT, which showed that 565 persons had NGT, 336 had isolated IFG (i-IFG), 72 had isolated IGT (i-IGT), 151 had combined IFG-IGT (IFG-IGT) and 78 had n-DM according to the criteria of the ADA [28]. All-cause and cardiovascular mortality were assessed over 7 years. Cardiovascular mortality was defined by ICD-9 codes 390-459 (diseases of the circulatory system) and code 798 (sudden death, cause unknown).

Oral glucose tolerance test OGTTs were carried out in the morning (7:00-11:00 hours) as previously described [27]. Fasting venous blood glucose was sampled and $75 \mathrm{~g}$ of anhydrous glucose (Dextro OGT, Boehringer, Mannheim, Germany) was given.

Laboratory analyses Blood was collected with minimal stasis, refrigerated at $4-8^{\circ} \mathrm{C}$ and shipped in refrigerant packaging within $2-4 \mathrm{~h}$ to the laboratory of Augsburg Central Hospital. Blood glucose was measured using a hexokinase method (Gluco-quant, Roche Diagnostics, Mannheim, Germany). $\mathrm{HbA}_{1 \mathrm{c}}$ was assessed using a turbidimetric immunological assay (Tina-quant, Roche Diagnostics). Total cholesterol was measured using the CHOD-PAP (Roche Diagnostics) and HDL-cholesterol using the phosphotungstic acid method (Boehringer). Triacylglycerols were measured using the GPO-PAP assay (nonfasting conditions in participants with diabetes) [27].

Anthropometric measurements and interviews Waist circumference was measured at the minimum abdominal girth. Blood pressure was measured three times as previously described $[20,29]$; the mean of the second and third measurement was used for the analysis. Hypertension was defined by $\geq 160$ / $\geq 95 \mathrm{mmHg}$ or known hypertension treated by antihypertensive agents. Medical history was assessed in a structured interview and included the use of prescription drugs. Regular smoking, high alcohol intake and physical activity level were defined as previously reported [29, 30]. Cardiovascular disease was defined as the need for hospital treatment of myocardial infarction and/or stroke [29, 30]. The metabolic syndrome was defined by the revised Adult Treatment Panel III criteria [31].
Classification of medications with possible influence on $H R V$ Drug classes that potentially increase HRV (positive effect) included angiotensin-converting enzyme inhibitors, angiotensin II (type 1) receptor blockers, $\beta 1$-selective $\beta$ receptor blockers without intrinsic sympathomimetic activity (ISA), nonselective $\beta$-receptor blockers without ISA (carvedilol), digitalis glycosides and parasympathomimetic and sympatholytic drugs. Drug classes that potentially decrease HRV (adverse effect) included $\beta 1$-selective $\beta$-receptor blockers with ISA, nonselective $\beta$-receptor blockers with ISA, tricyclic antidepressants, serotonin noradrenaline reuptake inhibitors (SNRIs; venlafaxine), selective serotonin reuptake inhibitors (SSRIs), anticholinergic and sympathomimetic drugs, and typical and atypical neuroleptics. Agents with no clear effect on HRV (neutral) included calcium channel blockers, nonselective $\beta$-receptor blockers without ISA and class III antiarrhythmics (sotalol).

Heart rate variability ECGs (lead II and lead V2 simultaneously) were recorded in the supine resting position over a period of $5 \mathrm{~min}$ (sample frequency $500 \mathrm{~Hz}$ ) as previously described [32]. In brief, time series of heart rate (tachograms) consisting of beat-to-beat intervals ( $\mathrm{R}-\mathrm{R}$ intervals) were extracted from the 5 min ECG recordings. Ectopic beats (ventricular or supra ectopic beats) and artefacts within the tachograms were detected and replaced by interpolated 'normal' beats by applying an adaptive filter to generate normal-to-normal $(\mathrm{NN})$ interval time series [33]. A total number of $120 \mathrm{HRV}$ variables (time domain, 15 indices; frequency domain, 15 indices; NLD, 90 indices using eight different methods) were determined by applying linear and nonlinear HRV analysis methods to the filtered tachograms. Calculations of the HRV indices were performed containing an in-house software package [34-36].

Linear HRV analysis For a detailed description, see previous reports $[32,37]$.

Time domain This involved determining the mean NN interval, SD of NN, CV of NN, SD of NN averages in $1 \mathrm{~min}$ segments (SDANN1), root mean squared successive difference (RMSSD), pNN50 (number of NN $>50 \mathrm{~ms} /$ number of all NN), pNN100, pNN200, pNN110 (portion of NN differences $<10 \mathrm{~ms}$ in all NN), pNN120, pNNl30, pNN150, Renyi2 (Renyi entropy of the histogram with [order] $\alpha=2$ ), Renyi4, Renyi0.25 and Shannon entropy.

Frequency domain This involved Fast Fourier transformation of the Blackman Harris window included ultra low frequency (ULF), very low frequency (VLF), low frequency (LF) and high frequency (HF); total power (TP); the ratios LF/HF, LF/ TP, HF/TP, VLF/TP, ULF/TP, ULF+VLF+LF/TP and ULF+ 
VLF/TP; the sum of ULF+VLF+LF; and LF-normalised units (NUs) and HFNUs.

Nonlinear HRV analysis For a detailed description, see the previous report [32].

Symbolic dynamics This involved determining the FWShannon (Shannon entropy of the word distribution [WD]), Forbword (forbidden words: number of seldom or never occurring word types with $p<0.001$ ), wpsum02 (relative portion (sum/total) of words consisting only of the symbols ' 0 ' and '2'), wpsum13, wsdvar (SD of the word sequence), plvar2 (portion of low-variability NN patterns $<2 \mathrm{~ms}$ ), phvar2 (portion of high-variability NN patterns $<2 \mathrm{~ms}$ ), plvar5, phvar5, plvar10, phvar10, plvar20, phvar20, FWRenyi0.25 (Renyi entropy of the word distribution with $\alpha=0.25)$ and FWRenyi4.

Short-term symbolic dynamics This involved determining the mis_pattern, $0 \mathrm{~V}, 1 \mathrm{~V}, 2 \mathrm{~V}$, auf, ab, huegel, tal, plateau, 2LV, 2UV and their SDs.

Detrended fluctuation analysis This involved determining the BBI $\alpha 1 \_4 \_16$ (short-term $[\alpha 1]$ fractal scaling exponent of detrended fluctuation analysis [DFA] slope $[\alpha]$ on a $\log -\log$ plot over particular regions of segment length $4-16$ beats) and the BBI $\alpha 2$ 16_64 (intermediate-term $[\alpha$ 2] fractal scaling exponent of DFA slope $[\alpha]$ on a loglog plot over particular regions of segment length 16-64 beats).

Compression entropy This involved determining compression (LZ77 routine) of the NN series with a window size of 3 and a lookahead buffer size of 3 (LZ77W3/LAB3), LZ77W5/LAB5 and LZ77W15/LAB5.

Auto-mutual information This involved determining a21RR (slope of the auto-transformation function [ati] from the maximum of ati $[\operatorname{tau}=0]$ to the subsequent value $[\operatorname{tau}=1])$ and a31RR $(\operatorname{tau}=2)$.

Auto-correlation function This involved determining a21RRcor (slope of the auto-correlation function [acor]) from the maximum of acor $(\mathrm{tau}=0)$ to the subsequent value ( $\mathrm{tau}=$ 1), a31RRcor ( $\operatorname{tau}=2$ ), $x 2$ peakRRcor (location of the greatest auxiliary maximum of the auto-correlation function), y2peakRRcor (amplitude) and amax21RRcor (slope of the auto-correlation function [acor] from the maximum of acor to the greatest auxiliary maximum of acor).

Poincaré plot The SD of the short axis (SDSA) and the SD of the long axis (SDLA) of the Poincare plot were determined, and used to calculate the SDSA/SDLA ratio.
Segmented Poincaré plot analysis This included 27 indexes, as previously described [32].

QT interval variability This was determined using the calculation: $\mathrm{QTVI}=\log 10\left[\left(\mathrm{QTV} / \mathrm{QTm}{ }^{2}\right) /\left(\mathrm{NNv} / \mathrm{NN}\right.\right.$ mean $\left.\left.^{2}\right)\right]$ [25].

Statistical analysis Continuous data were expressed by the median (interquartile range). Differences between groups were analysed using the Wilcoxon test. Qualitative data were described by frequency tables with $95 \%$ CIs and analysed using Fisher's exact test. To reduce skewness and to approximate normality, Yeo-Johnson transformation was used for the regression of the HRV variables and QTVI with age, sex, BMI and drugs potentially increasing or decreasing HRV and those without a clear effect on HRV [38]. The resulting regression line was used to define the lower and upper limits of normal for all HRV indices and QTVI at the 5th and 95th percentile of the NGT population, respectively. Since strong correlations were found between some HRV variables, one of us (A. Voss) selected 62 indices from the original 120 indices for the primary analysis. The rates of abnormal indices $(<5$ th or $>95$ th percentile) were compared between the NGT and k-DM groups using the Wilcoxon test. $P$ values were adjusted for multiple comparisons using Bonferroni correction. The approach of $\geq$ two abnormal HRV variables out of four indices from four different HRV classes was used as the primary analysis to estimate the prevalence of CAND in the six groups studied. The corresponding $p$ values were also corrected using the Bonferroni method. Multivariate linear regression analysis was performed to examine which risk factors are independently associated with the indices of HRV and QT variability as dependent variables. The latter were transformed using the Yeo-Johnson transformation [35] to account for skewness and approximate normality, followed by a stepwise model selection, starting with the full model and minimising the Akaike information criterion. Independent variables in the full model included age, sex, BMI, waist circumference, heart rate, physical activity, smoking, alcohol intake, dietary habits, hypertension, cardiovascular disease, drugs potentially increasing or decreasing HRV and those without a clear effect on HRV (3 groups), glucose tolerance status, $\mathrm{HbA}_{1 \mathrm{c}}$, serum triacylglycerols, HDL-cholesterol, LDL-cholesterol, creatinine and uric acid. Logistic models including risk factors identical to those remaining in the multivariate linear regression end models for HRV indices $<5$ th percentile including the AUC with asymptotic 95\% CIs, sensitivity, specificity, and positive and negative predictive values (PPV, NPV) were computed to obtain a screening score for CAND. All statistical tests were two-sided, and significance was set at $\alpha=0.05$. 


\section{Results}

Demographic and clinical characteristics of the six groups studied are shown in Table 1. BMI, waist circumference, triacylglycerols, and the rates of hypertension and the metabolic syndrome were higher in all other groups than in the NGT group. Age and the proportions of participants taking antihypertensive agents, drugs without a clear effect on HRV or those which may increase HRV were higher in the i-IGT, IFG-IGT, $\mathrm{n}$-DM, and k-DM groups than in the NGT group. The proportion of males was higher, $\mathrm{HbA}_{1 \mathrm{c}}$ was higher and HDLcholesterol was lower in the i-IFG, IFG-IGT, n-DM and kDM groups than in the NGT group. Heart rate was higher in the IFG-IGT, n-DM and k-DM groups than in the NGT group. LDL-cholesterol was higher in the k-DM group and serum creatinine was higher in the $\mathrm{i}-\mathrm{IFG}$ and IFG-IGT groups than in the NGT group. Compared with the NGT group, the proportion of regular smokers was lower in the i-IGT group, the level of physical activity was lower in the n-DM and kDM groups, prior hospitalisations due to myocardial infarction were more common in the k-DM group and the rates of participants taking drugs that may reduce HRV were lower in the i-IFG group. The proportion of individuals treated with statins was higher in the k-DM group than in the NGT group. No significant differences between the NGT group and all other groups studied were noted for high alcohol intake and prior hospitalisations due to stroke. Among participants with k-DM, 71.5\% received pharmacotherapy, 56.1\% were treated by oral glucose-lowering drugs and $23.1 \%$ were treated by insulin. All-cause and cardiovascular mortality were higher in the k-DM group than the NGT group; all-cause mortality was also higher in the n-DM group than the NGT group.

Table 1 Demographic and clinical characteristics of the six groups studied $(N=1,332)$

\begin{tabular}{|c|c|c|c|c|c|c|}
\hline Characteristic & NGT & i-IFG & i-IGT & IFG-IGT & n-DM & $\mathrm{k}-\mathrm{DM}$ \\
\hline$n$ & 565 & 336 & 72 & 151 & 78 & 130 \\
\hline Sex $(\% \text { male })^{\mathrm{a}}$ & $41.9(38.5,45.5)$ & $61.9(57.3,66.3)^{*}$ & $38.8(29.2,49.2)$ & $59.6(52.6,66.3)^{*}$ & $57.6(47.7,67.2)^{*}$ & $53.8(46.3,61.3)^{*}$ \\
\hline Age (years) ${ }^{\mathrm{b}}$ & $63(58-68)$ & $63(59-68)$ & $65(62-69)^{*}$ & $65(61-70)^{*}$ & $66(61-71)^{*}$ & $65(61-69)^{*}$ \\
\hline BMI $\left(\mathrm{kg} / \mathrm{m}^{2}\right)^{\mathrm{b}}$ & $26.9(24.7-29.5)$ & $28.2(25.8-30.8)^{*}$ & $29.4(26.1-32.0)^{*}$ & $29.1(27.4-31.9)^{*}$ & $29.9(27.9-33.0)^{*}$ & $31.2(28.1-34.3)^{*}$ \\
\hline Waist circumference $(\mathrm{cm})^{\mathrm{b}}$ & $92(85-99)$ & $98(91-104)^{*}$ & $96(89-102)^{*}$ & $100(94-108)^{*}$ & $102(96-112)^{*}$ & $102(97-110)^{*}$ \\
\hline Heart rate $(\mathrm{bpm})^{\mathrm{b}}$ & $67.1(61.9-73.1)$ & $67.9(61.8-75.5)$ & $68.7(62.3-74.3)$ & $68.5(62.6-75.3)^{*}$ & $71.0(63.7-81.1)^{*}$ & $68.7(63.5-76.9)^{*}$ \\
\hline Hypertension (\%) ${ }^{\mathrm{a}}$ & $28.0(24.7,31.2)$ & $36.0(31.7,40.5)^{*}$ & $52.8(42.5,62.9)^{*}$ & $55.0(47.9,61.8)^{*}$ & $60.3(50.3,69.6)^{*}$ & $70.8(63.5,77.3)^{*}$ \\
\hline Triacylglycerols $(\mathrm{mmol} / \mathrm{l})^{\mathrm{b}}$ & $1.16(0.84-1.62)$ & $1.33(0.98-1.81)^{*}$ & $1.30(1.03-1.85)^{*}$ & $1.45(1.16-2.11)^{*}$ & $1.65(1.30-2.25)^{*}$ & $2.23(1.53-3.15)^{*}$ \\
\hline HDL-cholesterol $(\mathrm{mmol} / \mathrm{l})^{\mathrm{b}}$ & $1.53(1.30-1.83)$ & $1.43(1.19-1.73)^{*}$ & $1.47(1.23-1.72)$ & $1.33(1.14-1.67)^{*}$ & $1.32(1.05-1.60)^{*}$ & $1.23(1.04-1.44) *$ \\
\hline LDL-cholesterol $(\mathrm{mmol} / \mathrm{l})^{\mathrm{b}}$ & $3.91(3.26-4.64)$ & $3.99(3.32-4.58)$ & $4.04(3.16-4.77)$ & $4.12(3.44-4.69)$ & $3.76(3.08-4.45)$ & $3.70(3.03-4.35)^{*}$ \\
\hline Creatinine $(\mu \mathrm{mol} / \mathrm{l})^{\mathrm{b}}$ & $72.5(64.5-83.1)$ & $77.8(68.1-86.6)^{*}$ & $71.6(65.4-83.1)$ & $77.8(68.1-88.4)^{*}$ & $76.0(68.1-88.4)$ & $76.0(65.4-86.6)$ \\
\hline $\mathrm{HbA}_{1 \mathrm{c}}(\%)^{\mathrm{b}}$ & $5.5(5.3-5.8)$ & $5.6(5.4-5.8)^{*}$ & $5.5(5.3-5.8)$ & $5.7(5.5-6.0)^{*}$ & $6.1(5.7-6.4)^{*}$ & $6.8(6.2-7.9)^{*}$ \\
\hline $\mathrm{HbA}_{1 \mathrm{c}}(\mathrm{mmol} / \mathrm{mol})^{\mathrm{b}}$ & $37(34-40)$ & $38(36-40)^{*}$ & $37(34-40)$ & $39(37-42)^{*}$ & $43(39-46)^{*}$ & $51(44-63)^{*}$ \\
\hline Regular smokers $(\%)^{\mathrm{a}}$ & $13.6(11.3,16.2)$ & $11.0(8.3,14.2)$ & $4.2(1.1,10.4)^{*}$ & $11.3(7.3,16.4)$ & $19.2(12.2,28.1)$ & $11.5(7.2,17.2)$ \\
\hline High alcohol intake $(\%)^{\mathrm{a}}$ & $18.0(15.4,20.9)$ & $22.9(19.2,27.0)$ & $19.4(12.2,28.7)$ & $23.8(18.2,30.2)$ & $25.6(17.7,35.0)$ & $13.8(9.1,19.8)$ \\
\hline Physically active (\%) ${ }^{\mathrm{a}}$ & $45.0(41.5,48.5)$ & $46.1(41.5,50.8)$ & $41.7(31.8,52.0)$ & $37.7(31.2,44.7)$ & $33.3(24.5,43.1)^{*}$ & $30.0(23.4,37.3)^{*}$ \\
\hline \multicolumn{7}{|l|}{ Prior hospitalisation due to } \\
\hline Myocardial infarction (\%) ${ }^{\mathrm{a}}$ & $3.7(2.5,5.3)$ & $3.3(1.8,5.4)$ & $1.4(0.1,6.4)$ & $5.3(2.3,9.4)$ & $6.4(2.6,13.0)$ & $10.8(6.6,16.3)^{*}$ \\
\hline Stroke $(\%)^{\mathrm{a}}$ & $1.9(1.1,3.2)$ & $1.8(0.8,3.5)$ & $5.6(1.9,12.3)$ & $4.0(1.7,7.7)$ & $3.8(1.1,9.6)$ & $3.1(1.1,6.9)$ \\
\hline Metabolic syndrome ATP III (\%) ${ }^{\mathrm{a}}$ & $15.0(12.6,17.7)$ & $53.9(49.2,58.5)^{*}$ & $30.6(21.7,40.7)^{*}$ & $72.8(66.3,78.8)^{*}$ & $84.6(76.3,90.9)^{*}$ & $87.7(81.9,92.1)^{*}$ \\
\hline \multicolumn{7}{|l|}{ Drugs that: } \\
\hline Reduce HRV (\%) ${ }^{\mathrm{a}}$ & $4.9(3.5,6.7)$ & $1.5(0.6,3.1)^{*}$ & $2.8(0.5,8.5)$ & $2.7(0.9,6.0)$ & $3.9(1.1,9.6)$ & $6.2(3.1,10.8)$ \\
\hline Do not change HRV $(\%)^{\mathrm{a}}$ & $6.2(4.6,8.1)$ & $7.4(5.2,10.2)$ & $16.6(9.9,25.6)^{*}$ & $15.3(10.6,20.9)^{*}$ & $11.6(6.2,19.3)^{*}$ & $14.7(9.8,20.7)^{*}$ \\
\hline Increase HRV $(\%)^{\mathrm{a}}$ & $21.4(18.6,24.5)$ & $26.1(22.3,30.4)$ & $36.1(26.7,46.4)^{*}$ & $35.8(29.3,42.7)^{*}$ & $34.6(25.7,44.5)^{*}$ & $46.9(39.4,54.5) *$ \\
\hline Statins $(\%)^{\mathrm{a}}$ & $8.1(6.3,10.3)$ & $7.4(5.2,10.2)$ & $12.5(6.7,20.8)$ & $12.6(8.4,17.9)$ & $11.5(6.2,19.3)$ & $17.7(12.4,24.1)^{*}$ \\
\hline Antihypertensive drugs $(\%)^{\mathrm{a}}$ & $27.1(24.0,30.3)$ & $30.1(25.9,34.4)$ & $48.6(38.4,58.9)^{*}$ & $51(44.0,58.0)^{*}$ & $44.9(35.2,54.8)^{*}$ & $63.1(55.6,70.1)^{*}$ \\
\hline All-cause mortality $(\%)^{\mathrm{a}}$ & $5.5(4.0,7.3)$ & $6.0(4.0,8.5)$ & $6.9(2.8,14.0)$ & $8.6(5.2,13.3)$ & $21.8(14.4,30.9)^{*}$ & $12.3(7.9,18.1)^{*}$ \\
\hline Cardiovascular mortality (\%) ${ }^{\mathrm{a}}$ & $2.5(1.5,3.8)$ & $1.8(0.8,3.5)$ & $2.8(0.5,8.5)$ & $3.3(1.3,6.8)$ & $5.1(1.8,11.4)$ & $6.2(3.1,10.8)^{*}$ \\
\hline
\end{tabular}

$* p<0.05$ vs NGT

${ }^{\text {a }}$ Data represent percentages $(95 \% \mathrm{CIs})$

${ }^{\mathrm{b}}$ Data represent median (1st to 3 rd quartiles) 
After Bonferroni correction, 17 out of $62 \mathrm{HRV}$ indices were more frequently abnormal in the k-DM group than the NGT group. These rates and those for QTVI with 95\% CIs are listed in Table 2. Only Renyi4 and TP showed higher rates of abnormal values in the i-IFG, IFG-IGT and n-DM groups than the NGT group. RMSSD, LF power and SDSA were more frequently abnormal in the IFG-IGT and n-DM groups than the NGT group. SDLA was more frequently abnormal in the iIFG and IFG-IGT groups than the NGT group. The percentage of abnormal SDANN1 was higher in the i-IFG and n-DM groups than the NGT group, and the percentage of those with abnormal compression entropy (LZ77W3/LAB3) was higher only in the n-DM group compared with the NGT group. The rates of abnormal QTVI did not differ between the NGT and the other groups.

To obtain the prevalence of abnormalities for a combination of HRV indices, four single indices from four different HRV classes showing the best discriminatory value between the NGT and the other groups were selected including Renyi4 (time domain), TP (frequency domain), SDSA (Poincaré plot) and SD of the word sequence (symbolic dynamics).
The results of the degree (severity) of CAND computed on the basis of the number of abnormal indices from one to four are presented in Table 3. The approach of $\geq$ two out of four abnormal variables gave a useful estimate of the prevalence of CAND as $<5 \%$ in the NGT population. Using this definition, the prevalence of CAND increased from $4.5 \%$ in the NGT group to $17.5 \%$ in the k-DM group and was significantly higher in the i-IFG, IFG-IGT, n-DM and k-DM groups compared with the NGT group. The definition of $\geq$ one out of four indices being abnormal gave higher rates of abnormalities in the IFG-IGT, n-DM and k-DM groups than in the NGT group, but the rate of $10.7 \%$ in the NGT group was excessively high. The definition of $\geq$ three out of four abnormal indices gave higher rates of abnormalities only for the $\mathrm{n}$ DM and k-DM groups vs the NGT group, while the constellation of all four indices being abnormal gave a difference only for the k-DM group vs the NGT group.

The results of multiple linear regression analysis to define the risk factors associated with four HRV measures from four different classes and QTVI in the entire study population are shown in Table 4. Deterioration in all five indices was

Table 2 Percentages (95\% CIs) of abnormal indices of HRV and QT variability index

\begin{tabular}{|c|c|c|c|c|c|c|}
\hline Index & NGT $(n=565)$ & i-IFG $(n=336)$ & i-IGT $(n=72)$ & IFG-IGT $(n=151)$ & $\mathrm{n}-\mathrm{DM}(n=78)$ & $\mathrm{k}-\mathrm{DM}(n=130)$ \\
\hline \multicolumn{7}{|l|}{ Time domain } \\
\hline SDNN & $4.7(3.1,6.8)$ & $6.6(4.1,9.9)$ & $2.9(0.4,10.2)$ & $8.7(4.7,14.5)$ & $9.1(3.7,17.8)$ & $15.9(10,23.4)^{*}$ \\
\hline SDANN1 & $5.2(3.5,7.4)$ & $9.7(6.7,13.5)^{*}$ & $4.4(0.9,12.4)$ & $7.4(3.7,14.5)$ & $13.0(6.4,22.6)^{*}$ & $12.7(7.4,19.8)^{*}$ \\
\hline CVNN & $4.5(3.0,6.6)$ & $4.7(2.7,7.6)$ & $4.4(0.9,12.4)$ & $4.7(1.9,9.4)$ & $6.5(2.1,14.5)$ & $9.5(5.0,16.5)^{*}$ \\
\hline RMSSD & $4.3(2.8,6.4)$ & $6.6(4.1,9.9)$ & $4.4(0.9,12.4)$ & $10.1(5.7,16.1)^{*}$ & $11.7(5.5,21)^{*}$ & $11.9(6.8,18.9)^{*}$ \\
\hline Renyi4 & $4.3(2.8,6.4)$ & $7.8(5.1,11.3)^{*}$ & $1.5(0,7.9)$ & $8.7(4.7,14.5)^{*}$ & $13.0(6.4,22.6)^{*}$ & $15.9(10,23.4)^{*}$ \\
\hline Shannon entropy & $4.7(3.1,6.8)$ & $6.9(4.4,10.2)$ & $2.9(0.4,10.2)$ & $8.7(4.7,14.5)$ & $9.1(3.7,17.8)$ & $16.7(10.6,24.3)^{*}$ \\
\hline \multicolumn{7}{|l|}{ Frequency domain } \\
\hline LF power & $5.2(3.5,7.4)$ & $8.1(5.4,11.7)$ & $7.4(2.4,16.3)$ & $10.1(5.7,16.1)^{*}$ & $14.3(7.4,24.1)^{*}$ & $15.9(10,23.4)^{*}$ \\
\hline HF power & $6.2(4.3,8.5)$ & $5.9(3.6,9.1)$ & $5.9(1.6,14.4)$ & $10.1(5.7,16.1)$ & $7.8(2.9,16.2)$ & $15.1(9.3,22.5)^{*}$ \\
\hline $\mathrm{TP}$ & $4.0(2.5,6)$ & $9.4(6.4,13.1)^{*}$ & $8.8(3.3,18.2)$ & $8.7(4.7,14.5)^{*}$ & $11.7(5.5,21)^{*}$ & $17.5(11.3,25.2)^{*}$ \\
\hline \multicolumn{7}{|l|}{ Symbolic dynamics } \\
\hline Shannon entropy WD & $4.5(3,6.6)$ & $5.6(3.4,8.7)$ & $4.4(0.9,12.4)$ & $6.7(3.3,12)$ & $3.9(0.8,11)$ & $12.7(7.4,19.8)^{*}$ \\
\hline Forbidden words & $5.8(4,8.1)$ & $5.9(3.6,9.1)$ & $7.4(2.4,16.3)$ & $8.7(4.7,14.5)$ & $7.8(2.9,16.2)$ & $14.3(8.7,21.6)^{*}$ \\
\hline SD word sequence & $6.2(4.3,8.5)$ & $6.9(4.4,10.2)$ & $7.4(2.4,16.3)$ & $10.1(5.7,16.1)$ & $7.8(2.9,16.2)$ & $15.9(10,23.4)^{*}$ \\
\hline Low variability $<20 \mathrm{~ms}$ & $1.1(0.4,2.4)$ & $0.0(0,1.2)$ & $1.5(0,7.9)$ & $0.7(0,3.7)$ & $2.6(0.3,9.1)$ & $4.8(1.8,10.1)^{*}$ \\
\hline Renyi E WD $\alpha=0.25$ & $7.6(5.5,10.1)$ & $6.6(4.1,9.9)$ & $7.4(2.4,16.3)$ & $8.7(4.7,14.5)$ & $7.8(2.9,16.2)$ & $15.9(10,23.4)^{*}$ \\
\hline \multicolumn{7}{|l|}{ Poincaré plot } \\
\hline SDSA & $4.3(2.8,6.4)$ & $6.6(4.1,9.9)$ & $4.4(0.9,12.4)$ & $10.1(5.7,16.1)^{*}$ & $11.7(5.5,21)^{*}$ & $11.9(6.8,18.9)^{*}$ \\
\hline SDLA & $4.5(3,6.6)$ & $8.4(5.6,12)^{*}$ & $1.5(0,7.9)$ & $9.4(5.2,15.3)^{*}$ & $9.1(3.7,17.8)$ & $16.7(10.6,24.3)^{*}$ \\
\hline \multicolumn{7}{|l|}{ Compression entropy } \\
\hline $\begin{array}{l}\text { LZ77 window=3, } \\
\text { lookahead buffer=3 }\end{array}$ & $4.9(3.2,7)$ & $5.3(3.1,8.4)$ & $5.9(1.6,14.4)$ & $6.7(3.3,12)$ & $11.7(5.5,21)^{*}$ & $15.1(9.3,22.5)^{*}$ \\
\hline \multicolumn{7}{|l|}{ QT variability } \\
\hline QTVI & $4.7(3.1,6.8)$ & $2.5(1.1,4.9)$ & $3.0(0.4,10.4)$ & $4.1(1.5,8.6)$ & $2.6(0.3,9.1)$ & $5.6(2.3,11.1)$ \\
\hline
\end{tabular}

${ }^{*} p<0.05$ vs NGT

CVNN, CV of NN; SDNN, SD of NN 
Table 3 Percentages (95\% CIs) of abnormal indices of HRV selected from four different HRV classes ${ }^{\mathrm{a}}$

\begin{tabular}{lllll}
\hline Participant group $(n)$ & \multicolumn{3}{l}{ Number of abnormal indices $(\%$ with $95 \%$ CI) } \\
\cline { 2 - 5 } & 4 out of 4 & $\geq 3$ out of 4 & $\geq 2$ out of 4 & $\geq 1$ out of 4 \\
\hline NGT (553) & $1.6(0.9,2.8)$ & $2.7(1.7,4.2)$ & $4.5(3.2,6.3)$ & $10.7(8.6,13.1)$ \\
i-IFG (320) & $1.3(0.4,2.8)$ & $4.1(2.4,6.4)$ & $8.1(5.8,11.1)^{\dagger}$ & $15.0(11.8,18.7)$ \\
i-IGT (68) & $1.5(0.1,6.8)$ & $2.9(0.5,9.0)$ & $5.9(2.0,13.0)$ & $16.2(9.3,25.4)$ \\
IFG-IGT (149) & $2.0(0.6,5.1)$ & $6.0(3.2,10.3)$ & $11.4(7.4,16.6)^{*}$ & $20.1(14.9,26.3)^{*}$ \\
n-DM (77) & $2.6(0.5,8.0)$ & $9.1(4.3,16.4)^{*}$ & $11.7(6.2,19.5)^{\dagger}$ & $22.1(14.6,31.3)^{*}$ \\
k-DM (126) & $6.4(3.2,11.2 *$ & $13.5(8.8,19.6)^{*}$ & $17.5(12.1,24.0)^{*}$ & $28.6(22.0,35.9)^{*}$ \\
\hline
\end{tabular}

${ }^{*} p<0.05$ vs NGT

${ }^{\dagger}$ Not significant after Bonferroni correction

${ }^{a}$ Renyi4 (time domain), TP spectrum (frequency domain), SD of short axis (Poincaré plot) and SD of the word sequence (symbolic dynamics)

associated with increasing heart rate and the presence of hypertension. Lower RMSSD, HF power, SDSA and compression entropy were associated with lower BMI and the use of drugs that may reduce HRV. Lower HF power and compression entropy were associated with increasing age, male sex and a higher $\mathrm{HbA}_{1 \mathrm{c}}$. Deterioration in RMSSD and SDSA was related to an increase in serum creatinine. Apart from heart rate and the presence of hypertension, a rise in QTVI was associated with an older age and lower physical activity. The association between three measures of HRV and smoking did not achieve statistical significance in the remaining models.

To obtain a screening score for the prediction of CAND in clinical practice, the variables included in the final models shown in Table 4 were entered into logistic models for HRV indices $<5$ th percentile. The highest AUC was obtained for RMSSD (0.86) and SDSA of the Poincaré plot (0.85; Table 5).
The corresponding intercepts were -10.7 and -10.9 . The sensitivity $(95 \% \mathrm{CI})$ of the screening score was $0.79(0.69,0.87)$ for RMSSD and $0.78(0.68,0.86)$ for SDSA, while the specificity was $0.81(0.79,0.83)$ and $0.83(0.80,0.85)$, respectively. The PPV was $0.23(0.18,0.28)$ for RMSSD and $0.25(0.20$, $0.30)$ for SDSA, while the corresponding NPV was 0.98 $(0.97,0.99)$ and $0.98(0.97,0.99)$, respectively.

Table 6 shows the 7 year all-cause and cardiovascular mortality in individuals with HRV indices from three different classes at $<5$ th percentile compared with those $\geq 5$ th percentile of the NGT population. All-cause mortality was significantly higher in individuals with $\mathrm{HRV}<5$ th percentile than in those $\geq 5$ th percentile, with the three indices showing identical results. Cardiovascular mortality was significantly higher in persons with Renyi4 and SDSA $<5$ th percentile than in those with Renyi4 and SDSA $\geq 5$ th percentile, while no such difference was noted for TP.

Table 4 Multiple linear regression analysis to define the risk factors associated with four HRV measures from four different classes and the QT variability index in the entire study population

\begin{tabular}{|c|c|c|c|c|c|c|c|c|c|c|}
\hline \multirow[t]{3}{*}{ Variable } & \multirow{2}{*}{\multicolumn{2}{|c|}{$\begin{array}{l}\text { Time domain } \\
\text { RMSSD }\end{array}$}} & \multirow{2}{*}{\multicolumn{2}{|c|}{$\begin{array}{l}\text { Frequency domain } \\
\text { HF power }\end{array}$}} & \multirow{2}{*}{\multicolumn{2}{|c|}{$\frac{\text { Poincaré plot }}{\text { SD short axis }}$}} & \multirow{2}{*}{\multicolumn{2}{|c|}{$\frac{\text { Compression entropy }}{\text { LZ77W3/LAB3 }}$}} & \multirow{2}{*}{\multicolumn{2}{|c|}{$\begin{array}{l}\text { QT interval variability } \\
\text { QTVI }\end{array}$}} \\
\hline & & & & & & & & & & \\
\hline & $\beta$ & $p$ value & $\beta$ & $p$ value & $\beta$ & $p$ value & $\beta$ & $p$ value & $\beta$ & $p$ value \\
\hline Heart rate (bpm) & -0.017 & $<0.001$ & -0.031 & $<0.001$ & -0.016 & $<0.001$ & -0.006 & $<0.001$ & 0.255 & $<0.001$ \\
\hline Age (years) & - & - & -0.011 & 0.005 & - & - & -0.003 & $<0.001$ & 0.146 & $<0.001$ \\
\hline Sex (female) & - & - & 0.128 & 0.002 & - & - & 0.011 & 0.081 & - & - \\
\hline BMI $\left(\mathrm{kg} / \mathrm{m}^{2}\right)$ & 0.005 & 0.015 & 0.007 & 0.154 & 0.005 & 0.020 & 0.001 & 0.075 & - & - \\
\hline Physically active (yes) & - & - & - & - & - & - & - & - & -0.067 & 0.011 \\
\hline Smoking (no) & 0.046 & 0.094 & 0.089 & 0.149 & 0.045 & 0.081 & - & - & - & - \\
\hline $\begin{array}{l}\text { Drugs with adverse effect } \\
\text { on HRV (yes) }\end{array}$ & -0.099 & 0.007 & -0.224 & 0.006 & -0.089 & 0.010 & -0.038 & 0.003 & - & - \\
\hline Hypertension (no) & 0.064 & $<0.001$ & 0.133 & 0.002 & 0.058 & 0.001 & 0.027 & $<0.001$ & -0.121 & $<0.001$ \\
\hline $\mathrm{HbA}_{1 \mathrm{c}}(\%)$ & - & - & -0.059 & 0.032 & - & - & -0.009 & 0.030 & - & - \\
\hline Serum creatinine $(\mu \mathrm{mol} / \mathrm{l})$ & -0.068 & 0.009 & - & - & -0.065 & 0.008 & - & - & - & - \\
\hline
\end{tabular}


Table 5 Screening score derived from logistic models for RMSSD and $\mathrm{SD}$ of Poincaré plot short axis $<5$ th percentile to predict cardiac autonomic dysfunction in clinical practice

\begin{tabular}{|c|c|c|c|c|}
\hline \multirow[t]{3}{*}{ Variable } & \multirow{2}{*}{\multicolumn{2}{|c|}{$\begin{array}{l}\text { Time domain } \\
\text { RMSSD }\end{array}$}} & \multirow{2}{*}{\multicolumn{2}{|c|}{$\frac{\text { Poincaré plot }}{\text { SDSA }}$}} \\
\hline & & & & \\
\hline & $\beta$ & $p$ value & $\beta$ & $p$ value \\
\hline Heart rate (bpm) & 0.130 & $<0.001$ & 0.128 & $<0.001$ \\
\hline Hypertension (no) & -0.838 & 0.001 & -0.812 & 0.002 \\
\hline BMI $\left(\mathrm{kg} / \mathrm{m}^{2}\right)$ & -0.026 & 0.365 & -0.016 & 0.584 \\
\hline Smoking (no) & -0.139 & 0.731 & -0.138 & 0.732 \\
\hline $\begin{array}{l}\text { Drugs with adverse } \\
\text { effect on HRV (yes) }\end{array}$ & -0.155 & 0.752 & -0.151 & 0.759 \\
\hline Serum creatinine $(\mu \mathrm{mol} / \mathrm{l})$ & 0.587 & 0.008 & 0.599 & 0.006 \\
\hline
\end{tabular}

\section{Discussion}

This study, conducted in the general population aged 5574 years, shows higher prevalence rates of CAND not only in individuals with known diabetes (17.5\%) but also in those with combined IFG-IGT (11.4\%) and n-DM (11.7\%) compared with the NGT group (4.5\%). The most useful novel HRV measure to differentiate between the NGT group and the five groups of glucose intolerance was Renyi4, which generally quantifies the diversity, uncertainty or randomness of a system. It is conceivable that Renyi4 was particularly sensitive because it considers both linear and nonlinear influences, which are characteristic of CAND. Among the nonlinear approaches, the Poincaré plot was more useful than compression entropy. The latter is another marker of complexity [39], but is more sensitive to nonlinear than linear variability. In contrast to Renyi4, compression entropy does not evenly consider both influences, which may explain its lower sensitivity (for further details, see electronic supplementary material $[\mathrm{ESM}])$. Finally, modifiable cardiovascular risk factors associated with reduced HRV, including heart rate, BMI, hypertension, smoking, serum creatinine and use of drugs suppressing HRV, can be integrated in a simple score to screen for CAND in clinical practice.
Nine population-based studies assessing the relationship between HRV and glucose intolerance have been published [8-16]. While some studies have demonstrated reduced measures of HRV or heart rate in persons with IFG compared with those with NFG [8-10], others showed no such differences [11-15]. Moreover, these studies did not evaluate the complete spectrum of glucose intolerance, i.e. either persons with IFG but not IGT were included [8-11, 13, 14] or vice versa $[15,16]$, or no NFG/NGT population was included [14] and participants with IFG/IGT and diabetes were mixed [14].

A novel finding of this study was the higher rate of abnormal HRV for six indices in the combined IFG-IGT group vs the NGT group. The former group shows the highest risk of progression to type 2 diabetes: 70.4 per 1,000 person-years, compared with 47.4 per 1,000 person-years for the IFG group and 45.5 per 1,000 person-years for the IGT group [40]. Thus, the IFG-IGT group is in jeopardy of additive risk arising from diabetes and autonomic dysfunction. Models for prevention policies on future diabetes prevalence in the USA suggest a 'high-risk' strategy for IFG-IGT persons, who should receive structured lifestyle intervention [41]. We also observed a higher rate of abnormal HRV for four indices in the i-IFG but not the i-IGT group compared with the NGT group. However, when CAND was defined by $\geq$ two abnormalities out of four HRV indices, Bonferroni correction abolished statistical significance for both of the comparisons i-IFG (8.1\%) vs NGT $(4.5 \%)$ and n-DM (11.7\%) vs NGT. However, we believe that these differences are clinically relevant, since the rates were 1.8-fold and 2.6-fold higher than in the NGT population, respectively.

The strengths of the present study are the population-based approach; the relatively large sample size; inclusion of the complete spectrum of glucose intolerance with the opportunity to discriminate between five different categories; the selection of the most useful measures from a comprehensive array of $120 \mathrm{HRV}$ indices derived from two linear and eight nonlinear techniques as well as QT variability; the use of normal limits for HRV adjusted for the most important confounders including drugs that may lower or increase HRV; and the detailed metabolic phenotyping. This study also has some limitations. First, the cross-sectional design does not provide

Table 6 All-cause and cardiovascular mortality over 7 years in participants with HRV indices from three different classes ${ }^{\mathrm{a}}$

\begin{tabular}{|c|c|c|c|c|c|c|c|c|c|c|}
\hline \multirow[t]{3}{*}{ HRV index } & \multicolumn{5}{|c|}{ All-cause mortality } & \multicolumn{5}{|c|}{ Cardiovascular mortality } \\
\hline & \multicolumn{2}{|c|}{$<5$ th percentile } & \multicolumn{2}{|c|}{$\geq 5$ th percentile } & \multirow[t]{2}{*}{$p$ value } & \multicolumn{2}{|c|}{$<5$ th percentile } & \multicolumn{2}{|c|}{$\geq 5$ th percentile } & \multirow[t]{2}{*}{$p$ value } \\
\hline & $n$ & $\%(95 \% \mathrm{CI})$ & $n$ & $\%(95 \% \mathrm{CI})$ & & $n$ & $\%(95 \% \mathrm{CI})$ & $n$ & $\%(95 \% \mathrm{CI})$ & \\
\hline Time domain: Renyi4 & $11 / 65$ & $16.9(9.8,26.5)$ & $88 / 1,230$ & $7.2(6.0,8.5)$ & 0.013 & $5 / 59$ & $8.5(3.4,17.0)$ & $33 / 1,175$ & $2.8(2.1,3.7)$ & 0.031 \\
\hline Frequency domain: TP & $11 / 65$ & $16.9(9.8,26.5)$ & $88 / 1,230$ & $7.2(6.0,8.5)$ & 0.013 & $4 / 58$ & $6.9(2.4,15.1)$ & $34 / 1,176$ & $2.9(2.1,3.8)$ & 0.099 \\
\hline Poincaré plot: SDSA & $11 / 65$ & $16.9(9.8,26.5)$ & $88 / 1,230$ & $7.2(6.0,8.5)$ & 0.013 & $5 / 59$ & $8.5(3.4,17.0)$ & $33 / 1,175$ & $2.8(2.1,3.7)$ & 0.031 \\
\hline
\end{tabular}

${ }^{a}<5$ th percentile compared with those $\geq 5$ th percentile of the NGT population 
any insight into causality and prediction, but this was not intended. Second, sample sizes were different between groups as a result of distribution in the population studied. Thus, the relatively low number of individuals included in the i-IGT and n-DM groups could represent a potential source of bias. Indeed, the i-IGT group somewhat differed from the other glucose intolerant groups inasmuch as it included a higher number of women and lower number of regular smokers, persons with the metabolic syndrome and those with previous hospitalisation due to myocardial infarction. For example, a recent meta-analysis reported that HRV is reduced in women with the metabolic syndrome compared with those without [42]. Taken together, we cannot rule out the possibility that these differences may have contributed to the relatively low percentage of abnormal HRV measures in the i-IGT group. Third, since the age range of the sample studied herein was 55-74 years, the study results may not be readily extrapolated to younger populations.

We also show that modifiable cardiovascular risk factors are associated with reduced HRV. These factors 'explained' $25 \%$ of the variance in compression entropy (LZ77W3/ LAB3) and $23 \%$ of the variance in RMSSD or SDSA. Hypertension was among the risk factors most consistently associated with low HRV and a higher degree of physical activity was associated with lower QTVI, which is considered a potential determinant of ventricular arrhythmias and cardiac mortality [43]. Therefore, antihypertensive treatment with agents that may augment vagal activity and reduce QT variability may be beneficial in patients with low HRV and high QT variability [44].

For practical purposes, we constructed a simple screening score comprising heart rate, BMI, hypertension, smoking, serum creatinine and the use of drugs suppressing HRV to predict the individual likelihood of diminished HRV. The highest AUC was obtained for RMSSD (0.86) and SDSA (0.85), with good sensitivity $(0.79 / 0.78)$, very good specificity $(0.81 / 0.83)$ and excellent NPV (0.98). Thus, particularly if the score result is negative, the presence of CAND can be ruled out with a high degree of certainty. A similar 'risk score' has recently been developed in a Chinese population, but its AUC (0.73), sensitivity (0.69) and specificity (0.78) were somewhat lower than ours [45]. Such simple screening scores could be used in clinical practice if equipment for measuring HRV is not readily available, but further validation is needed.

The associations between low HRV and modifiable cardiovascular risk factors observed herein also suggest that both could be amenable to lifestyle modification. In the Diabetes Prevention Program, lifestyle intervention in persons with IFG or IGT resulted in a decrease in heart rate and increase in HRV over 4 years [46]. An 8 week exercise intervention was associated with improvement in the components of the metabolic syndrome and HRV variables [47].
In conclusion, using a comprehensive array of different classes of HRV variables in the general population aged 5574 years, we demonstrated a higher prevalence of CAND in participants with $\mathrm{k}-\mathrm{DM}$ and n-DM, as well as a particularly higher prevalence in those with combined IFG-IGT and modest increase in persons with isolated IFG as opposed to those with isolated IGT. Reduced HRV was associated with higher mortality and modifiable cardiovascular risk factors. Strategies aimed at reducing cardiovascular risk by effective lifestyle intervention may exert favourable effects on autonomic dysfunction and presumably ultimately also on cardiovascular mortality.

Acknowledgements The authors wish to thank M. Scheer for conducting the statistical analysis, the staff at the KORA study centre for organising and conducting the data collection and all volunteers for participating in this study.

Funding The KORA research platform and the KORA Augsburg studies are financed by the Helmholtz Zentrum München, German Research Centre for Environmental Health, which is funded by the German Federal Ministry of Education and Research and the State of Bavaria. This work was supported by the Ministry of Science and Research of the State of North Rhine-Westphalia and the German Federal Ministry of Health.

Duality of interest All authors declare that there is no duality of interest associated with this manuscript.

Contribution statement DZ designed the study and wrote the manuscript; DZ, AV, SP, AS, AP, MR, WR and CM collected and analysed the data and revised the manuscript. All authors contributed substantially to the conception or design of the work or to the acquisition, analysis, or interpretation of the data; and to drafting the work or revising it critically for important intellectual content; gave final approval of the version to be published; and agreed to be accountable for all aspects of the work in ensuring that questions related to the accuracy or integrity of any part of the work are appropriately investigated and resolved. DZ is the guarantor of this work and, as such, had full access to all of the data in the study and takes responsibility for the integrity of the data and the accuracy of the data analysis.

\section{References}

1. Mellbin LG, Anselmino M, Rydén L (2010) Diabetes, prediabetes and cardiovascular risk. Eur J Cardiovasc Prev Rehabil 17(Suppl 1): S9-S14

2. The Task Force on diabetes, pre-diabetes, and cardiovascular diseases of the European Society of Cardiology (ESC) and developed in collaboration with the European Association for the Study of Diabetes (EASD), Rydén L, Grant PJ, Anker SD et al (2013) ESC Guidelines on diabetes, pre-diabetes, and cardiovascular diseases developed in collaboration with the EASD. Eur Heart J 34:3035-3087

3. Qiao Q, Pyörälä K, Pyörälä M et al (2002) Two-hour glucose is a better risk predictor for incident coronary heart disease and cardiovascular mortality than fasting glucose. Eur Heart J 23:1267-1275

4. Ning F, Tuomilehto J, Pyörälä K, Onat A, Soderberg S, Qiao Q (2010) Cardiovascular disease mortality in Europeans in relation to fasting and 2-h plasma glucose levels within a normoglycemic range. Diabetes Care 33:2211-2216 
5. Papanas N, Vinik AI, Ziegler D (2011) Neuropathy in prediabetes: does the clock start ticking early? Nat Rev Endocrinol 7:682-690

6. Ziegler D, Papanas N, Vinik AI, Shaw JE (2014) Epidemiology of polyneuropathy in diabetes and prediabetes. Handb Clin Neurol 126: 3-22

7. Vinik AI, Ziegler D (2007) Diabetic cardiovascular autonomic neuropathy. Circulation 115:387-397

8. Panzer C, Lauer MS, Brieke A, Blackstone E, Hoogwerf B (2002) Association of fasting plasma glucose with heart rate recovery in healthy adults: a population-based study. Diabetes 51:803-807

9. Schroeder EB, Chambless LE, Liao D et al (2005) Atherosclerosis Risk in Communities (ARIC) study. Diabetes, glucose, insulin, and heart rate variability: the Atherosclerosis Risk in Communities (ARIC) study. Diabetes Care 28:668-674

10. Stein PK, Barzilay JI, Domitrovich PP et al (2007) The relationship of heart rate and heart rate variability to non-diabetic fasting glucose levels and the metabolic syndrome: the Cardiovascular Health Study. Diabet Med 24:855-863

11. Singh JP, Larson MG, O'Donnell CJ et al (2000) Association of hyperglycemia with reduced heart rate variability: The Framingham Heart Study. Am J Cardiol 86:309-312

12. Wu JS, Yang YC, Lin TS et al (2007) Epidemiological evidence of altered cardiac autonomic function in subjects with impaired glucose tolerance but not isolated impaired fasting glucose. J Clin Endocrinol Metab 92:3885-3889

13. Intzilakis T, Hartmann G, Mouridsen MR et al (2013) Soluble urokinase plasminogen activator receptor. C-reactive protein and triglyceride are associated with heart rate variability in nondiabetic Danes. Eur J Clin Investig 43:457-468

14. Hsu WC, Yen AM, Liou HH, Wang HC, Chen TH (2009) Prevalence and risk factors of somatic and autonomic neuropathy in prediabetic and diabetic patients. Neuroepidemiology 33:344-349

15. Gerritsen J, Dekker JM, TenVoorde BJ et al (2000) Glucose tolerance and other determinants of cardiovascular autonomic function: the Hoorn Study. Diabetologia 43:561-570

16. Fujimoto WY, Leonetti DL, Kinyoun JL, Shuman WP, Stolov WC, Wahl PW (1987) Prevalence of complications among secondgeneration Japanese-American men with diabetes, impaired glucose tolerance, or normal glucose tolerance. Diabetes 36:730-739

17. Perciaccante A, Fiorentini A, Paris A, Serra P, Tubani L (2006) Circadian rhythm of the autonomic nervous system in insulin resistant subjects with normoglycemia, impaired fasting glycemia, impaired glucose tolerance, type 2 diabetes mellitus. BMC Cardiovase Disord 6:19

18. Isak B, Oflazoglu B, Tanridag T, Yitmen I, Us O (2008) Evaluation of peripheral and autonomic neuropathy among patients with newly diagnosed impaired glucose tolerance. Diabetes Metab Res Rev 24: 563-569

19. Diakakis GF, Parthenakis FI, Patrianakos AP et al (2008) Myocardial sympathetic innervation in patients with impaired glucose tolerance: relationship to subclinical inflammation. Cardiovasc Pathol 17:172177

20. Elming MB, Hornum M, Feldt-Rasmussen B, Mathiesen ER (2011) Cardiac autonomic neuropathy in patients with uraemia is not related to pre-diabetes. Dan Med Bull 58:A4244

21. Laitinen T, Lindström J, Eriksson J et al (2011) Cardiovascular autonomic dysfunction is associated with central obesity in persons with impaired glucose tolerance. Diabet Med 28:699-704

22. Thiyagarajan R, Subramanian SK, Sampath N et al (2012) Association between cardiac autonomic function, oxidative stress and inflammatory response in impaired fasting glucose subjects: cross-sectional study. PLoS One 7:e41889

23. Putz Z, Németh N, Istenes I et al (2013) Autonomic dysfunction and circadian blood pressure variations in people with impaired glucose tolerance. Diabet Med 30:358-362
24. Voss A, Schulz S, Schroeder R, Baumert M, Caminal P (2009) Methods derived from nonlinear dynamics for analysing heart rate variability. Philos Trans A Math Phys Eng Sci 367:277-296

25. Berger RD, Kasper EK, Baughman KL, Marban E, Calkins H, Tomaselli GF (1997) Beat-to-beat QT interval variability: novel evidence for repolarization lability in ischemic and nonischemic dilated cardiomyopathy. Circulation 96:1557-1565

26. Dobson CP, Kim A, Haigney M (2013) QT variability index. Prog Cardiovasc Dis 56:186-194

27. Rathmann W, Haastert B, Icks A et al (2003) High prevalence of undiagnosed diabetes mellitus in Southern Germany: target populations for efficient screening. The KORA survey 2000. Diabetologia 46:182-189

28. American Diabetes Association (2014) Diagnosis and classification of diabetes mellitus. Diabetes Care 37(Suppl 1):S81-S90

29. Ziegler D, Zentai C, Perz S et al (2006) Selective contribution of diabetes and other cardiovascular risk factors to cardiac autonomic dysfunction in the general population. Exp Clin Endocrinol Diabetes 114:153-159

30. Ziegler D, Zentai CP, Perz S et al (2008) Prediction of mortality using measures of cardiac autonomic dysfunction in the diabetic and nondiabetic population: the MONICA/KORA Augsburg Cohort Study. Diabetes Care 31:556-561

31. Grundy SM, Cleeman JI, Daniels SR et al (2005) American Heart Association; National Heart, Lung, and Blood Institute. Diagnosis and management of the metabolic syndrome: an American Heart Association/National Heart, Lung, and Blood Institute Scientific Statement. Circulation 112:2735-2752

32. Voss A, Schroeder R, Fischer C, Heitmann A, Peters A, Perz S (2013) Influence of age and gender on complexity measures for short term heart rate variability analysis in healthy subjects. Conf Proc IEEE Eng Med Biol Soc 2013:5574-5577

33. Wessel N, Voss A, Malberg H et al (2000) Nonlinear analysis of complex phenomena in cardiological data Z. Herzschrittmacherther Elektrophysiol 11:159-173

34. Voss A, Schroeder R, Vallverdú M et al (2013) Short-term vs. longterm heart rate variability in ischemic cardiomyopathy risk stratification. Front Physiol 4:364

35. Voss A, Heitmann A, Schroeder R, Peters A, Perz S (2012) Shortterm heart rate variability - age dependence in healthy subjects. Physiol Meas 33:1289-1311

36. Voss A, Goernig M, Schroeder R, Truebner S, Schirdewan A, Figulla HR (2012) Blood pressure variability as sign of autonomic imbalance in patients with idiopathic dilated cardiomyopathy. Pacing Clin Electrophysiol 35:471-479

37. Task Force of the European Society of Cardiology and the North American Society of Pacing and Electrophysiology (1996) Heart rate variability: standards of measurement, physiological interpretation and clinical use. Circulation 93:1043-1065

38. Yeo IK, Johnson R (2000) A new family of power transformations to improve normality or symmetry. Biometrika 87:954-959

39. Baumert M, Baier V, Haueisen J et al (2004) Forecasting of life threatening arrhythmias using the compression entropy of heart rate. Methods Inf Med 43:202-206

40. Morris DH, Khunti K, Achana F et al (2013) Progression rates from $\mathrm{HbA}_{1 \mathrm{c}} 6.0-6.4 \%$ and other prediabetes definitions to type 2 diabetes: a meta-analysis. Diabetologia 56:1489-1493

41. Gregg EW, Boyle JP, Thompson TJ, Barker LE, Albright AL, Williamson DF (2013) Modeling the impact of prevention policies on future diabetes prevalence in the United States: 2010-2030. Popul Health Metrics 11:18

42. Stuckey MI, Tulppo MP, Kiviniemi AM, Petrella RJ (2014) Heart rate variability and the metabolic syndrome: a systematic review of the literature. Diabetes Metab Res Rev 30:784-793

43. Niemeijer MN, van den Berg ME, Eijgelsheim M et al (2014) Shortterm QT variability markers for the prediction of ventricular 
arrhythmias and sudden cardiac death: a systematic review. Heart 100:1831-1836

44. Galetta F, Franzoni F, Fallahi P, Tocchini L, Graci F, Carpi A, Antonelli A, Santoro G (2009) Effect of telmisartan on QT interval variability and autonomic control in hypertensive patients with left ventricular hypertrophy. Biomed Pharmacother 64:516-520

45. Ge X, Pan SM, Zeng F, Tang ZH, Wang YW (2014) A simple Chinese risk score model for screening cardiovascular autonomic neuropathy. PLoS One 9:e89623
46. Carnethon MR, Prineas RJ, Temprosa M, Zhang ZM, Uwaifo G, Molitch ME (2006) Diabetes Prevention Program Research Group. The association among autonomic nervous system function, incident diabetes, and intervention arm in the Diabetes Prevention Program. Diabetes Care 29:914-919

47. Stuckey MI, Kiviniemi AM, Petrella RJ (2013) Diabetes and technology for increased activity study: the effects of exercise and technology on heart rate variability and metabolic syndrome risk factors. Front Endocrinol (Lausanne) 4:121 